\title{
On the central ionizing star of G23.96+0.15 and near-IR spectral classification of $O$ stars (Research Note)
}

\author{
P. A. Crowther and J. P. Furness ${ }^{\star}$
}

\author{
Department of Physics \& Astronomy, University of Sheffield, Hounsfield Road, Sheffield, S3 7RH, UK \\ e-mail: Paul.Crowther@sheffield.ac.uk
}

Received 9 September 2008 / Accepted 14 October 2008

ABSTRACT

\begin{abstract}
Aims. A near-infrared study of the main ionizing star of the ultracompact HII region G23.96+0.15 (IRAS 18317-0757) is presented, along with a re-evaluation of the distance to this source, and a re-assessment of $H$-and $K$-band classification diagnostics for $\mathrm{O}$ dwarfs Methods. We have obtained near-IR VLT/ISAAC imaging and spectroscopy of G23.96+0.15, plus archival imaging from UKIRT/UFTI. A spectroscopic analysis was carried out using a non-LTE model atmosphere code.

Results. A quantitative $H$ - and $K$-band classification scheme for $\mathrm{O}$ dwarfs is provided, from which we establish an $\mathrm{O} 7.5 \mathrm{~V}$ spectral subtype for the central star of G23.96+0.15. We estimate an effective temperature of $T_{\text {eff }} \sim 38 \mathrm{kK}$ from a spectral analysis.

Conclusions. A spectroscopic distance of $2.5 \mathrm{kpc}$ is obtained for G23.96+0.15, substantially lower than the kinematic distance of $4.7 \mathrm{kpc}$, in common with recent studies of other Milky way H II regions. Such discrepancies would be alleviated if sources are unresolved binaries or clusters.
\end{abstract}

Key words. ISM: HII regions - ISM: dust, extinction - stars: early-type - stars: fundamental parameters

\section{Introduction}

The formation of high mass stars remains an unresolved problem in contemporary astrophysics (Zinnecker \& Yorke 2007). Despite considerable theoretical progress in recent years, this topic remains observationally challenging. O stars form deeply embedded within their natal cocoons, presumably within intermediate to high mass star clusters, only to visually emerge after $\sim 0.5 \mathrm{Myr}$ (Prescott et al. 2007), a substantial fraction of their 2-10 Myr main-sequence lifetimes.

Radio continuum surveys of ultracompact H II (UCHII) regions betray the presence of $\mathrm{O}$ stars through the effect of their Lyman continuum photons to free-free (thermal) emission. Typically, these regions can not be observed at wavelengths shorter than the mid-infrared, such that the ionizing stars can only be studied indirectly through the circumstellar dust and gas (e.g. Peeters et al. 2002; Martín-Hernández et al. 2002). However, in a few instances our sight line to the central source is sufficiently clear of circumstellar dust that the $\mathrm{O}$ star responsible for the $\mathrm{H}$ II region can be directly observed at near-infrared wavelengths (Watson \& Hanson 1997; Hanson et al. 2002; Bik et al. 2005).

Over the past decade, the advent of efficient near-IR spectrographs at large ground-based telescopes has permitted medium resolution $(R \sim 5000)$ spectroscopy of template Milky Way $\mathrm{O}$ stars in the $H$ - and $K$-bands (Hanson et al. 2005b), which has been extended to the O4-5 star responsible for the G29.96-0.02 UCHII region (IRAS 8434-0242, Hanson et al. 2005a). Spectroscopic analysis of near-IR hydrogen and helium

\footnotetext{
* Based on observations made with ESO telescopes at the Paranal Observatory under programme ID 077.C-0550(A).
}

lines of $\mathrm{O}$ stars agrees closely with optical diagnostics in most cases (Repolust et al. 2005).

Thus far, G29.96-0.02 represents the sole example of an UCHII region whose ionizing star has been analysed based upon medium spectral resolution. The significance of such objects is that, in principal, they may serve as calibrators for the so-called "inverse problem", in which the ionizing stars of embedded UCHII regions may be obtained from analysis of mid-infrared fine-structure nebular lines (e.g. Martín-Hernández et al. 2002; Morisset et al. 2004).

Fortunately, other UCHII regions are also accessible to near-IR spectroscopy. One such region, G23.96+0.15 (IRAS 18317-0757) is the focus of the present study, for which Hanson et al. (2002) estimated a spectral type of 07-8 from low resolution $K$-band spectroscopy and Kim \& Koo (2001) have inferred a mid-O spectral type from radio continuum observations for an adopted distance of 6 kpc. Hunter et al. (2004) have identified a cluster of embedded massive stars from millimetre observations of G23.96+0.15.

In the present study, we present new near-IR imaging and spectroscopy of G23.96+0.15 permitting the subtype of the ionizing star to be refined from $H$-band and $K$-band hydrogen and helium line ratios, plus a spectroscopic distance for comparison with kinematic results.

\section{Observations}

$H$-band and $K$-band spectroscopy of $\mathrm{G} 23.96+0.15$ was observed with the ISAAC instrument (Moorwood et al. 1998) mounted at the Very Large Telescope between 22 April-2 May 2006. High spatial resolution imaging was drawn from acquisition ISAAC datasets, which were supplemented by archival UKIRT Fast 

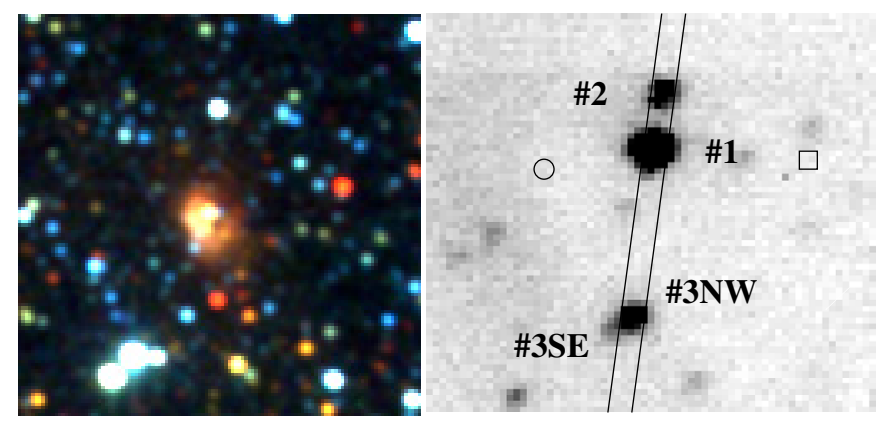

Fig. 1. (left) $2 \times 2$ arcmin composite $J H K$ image centered upon G23.96+0.15 from 2MASS; (right) $10 \times 10$ arcsec $\sim 2.18 \mu \mathrm{m}$ acquisition image of G23.96+0.15 from ISAAC, in which the brightest sources are marked with the ISAAC slit overlaid. Source \#1 has position $\alpha=18: 34: 25.25, \delta=-07: 54: 45.5$ (J2000), with the locations of the $6 \mathrm{~cm}$ peak from Wood \& Churchwell (1989, circle) and $21 \mathrm{~cm}$ peak from Kim \& Koo (2001, square) also indicated. North is up and east is to the left. (This figure is available in color in this electronic version.)

Trace Imager (UFTI, Roche et al. 2002) $K$-band imaging, plus $H$-band imaging from Hanson et al. (2002) and 2MASS datasets.

\subsection{Near-IR imaging}

Acquisition ISAAC images of G23.96+0.15 were obtained using the $1024 \times 1024$ Hawaii Rockwell array $(0.148 \mathrm{arcsec} / \mathrm{pix})$, with a combination of both the $2.17 \mu \mathrm{m}$ and $2.19 \mu \mathrm{m}$ narrowband filters during excellent seeing conditions of $0.3-0.5$ arcsec in April-May 2006.

In Fig. 1 we present a $2 \times 2$ arcmin $J H K$ composite image centered upon $\mathrm{G} 23.96+0.15$ from 2 MASS plus the central $10 \times 10$ arcsec of the $\sim 2.18 \mu$ m ISAAC dataset. The ISAAC image reveals a bright source (\#1), located at $\alpha=18: 34: 25.25$, $\delta=-07: 54: 45.5$ (J2000.0). This source lies $\sim 2.7$ arcsec west of the $6 \mathrm{~cm}$ peak reported by Wood \& Churchwell (1989), though 3.5 arcsec east of the $21 \mathrm{~cm}$ peak of Kim \& Koo (2001) for this irregular UCHII region. Fainter near-IR sources lie 1.5 arcsec to the north of \#1 and 4.2 arcsec to the south, which we shall refer to as sources \#2 and 3, respectively. Source \#3 is itself resolved into NW and SE components, separated by 0.7 arcsec. Photometry of these sources was obtained from archival UKIRT UFTI images from 19 Jul. 2000 obtained with the $1024 \times 1024$ Hawaii array (0.09 arcsec/pix) and $K$-band (K98) filter during seeing conditions of $\sim 0.6$ arcsec, using a zero-point obtained from 10 nearby 2 MASS field stars, providing an accuracy of $\pm 0.04 \mathrm{mag}$. The $J$-, $H$ - and $K$-band magnitudes for the combined sources \#1 and \#2 are 13.81, 11.46 and 9.94 mag, respectively (2MASS 18342523-0754455).

We have also inspected $H$-band images from the Steward $2.3 \mathrm{~m}$ IRCam guider images of Hanson et al. (2002) using a $128 \times 128$ Hawaii array $(0.5 \mathrm{arcsec} / \mathrm{pix})$, obtained in seeing conditions of $\sim 1.5$ arcsec, in which sources \#1 and \#2 are again blended, from which $H(\# 3)-H(\# 1+\# 2) \sim 1.5 \mathrm{mag}$. Independent estimates of $H(\# 3 \mathrm{NW})-H(\# 2) \sim 0.3 \mathrm{mag}$ and $H(\# 3 \mathrm{NW})-$ $H(\# 1) \sim 2.3$ mag have been obtained from our high spatial resolution ISAAC $1.71 \mu \mathrm{m}$ long-slit spectroscopy (see next section). These sources were well aligned, so slit losses should minimal. Photometric properties are presented in Table 1, taking into account uncertainties resulting from the ISAAC and IRCam observations.
Table 1. Near-IR properties of G23.96+0.15 sources from UKIRT/UFTI ( $K$-band) and Steward $2.3 \mathrm{~m} / \mathrm{IRCam}$ imaging $(H$-band) plus VLT/ISAAC acquisition images and spectroscopy, from which equivalenth widths ( $W_{\lambda}$ in $\AA$ ) were also measured for \#1.

\begin{tabular}{cccccccc}
\hline \hline Source & $K$ & $H-K$ & $W_{\lambda}(\mathrm{He}$ II $)$ & $W_{\lambda}(\mathrm{He} \mathrm{I})$ & $W_{\lambda}(\mathrm{HI})$ & $W_{\lambda}(\mathrm{He}$ II $)$ & Spect. \\
& Mag & $\mathrm{Mag}$ & $1.692 \mu \mathrm{m}$ & $1.700 \mu \mathrm{m}$ & $2.165 \mu \mathrm{m}$ & $2.189 \mu \mathrm{m}$ & Type \\
\hline$\# 1$ & 10.43 & 1.2 & 0.20 & 1.24 & 2.95 & 0.94 & O7.5V \\
& \pm 0.04 & \pm 0.1 & \pm 0.08 & \pm 0.11 & \pm 0.17 & \pm 0.12 & \\
$\# 2$ & 12.00 & 1.7 & & & & & \\
& \pm 0.04 & \pm 0.2 & & & & & \\
$\# 3 \mathrm{NW}$ & 12.10 & 1.9 & & & & & \\
& \pm 0.04 & \pm 0.3 & & & & & \\
$\# 3 S E$ & 13.7 & -0.2 & & & & & \\
& \pm 0.2 & \pm 0.3 & & & & & \\
\hline
\end{tabular}

\subsection{Near-IR spectroscopy}

Long-slit spectroscopy of G23.96+0.15 was obtained with ISAAC in April-May 2006 at a position angle of 7.7 degrees west of north, in order to include sources \#1, \#2 and \#3NW at three medium resolution, $0.775 \AA$ /pix) grating settings centered at 1.71, 2.09 and $2.20 \mu \mathrm{m}$ (recall Fig. 1). These observations, obtained using a 0.6 arcsec slit width, were taken during excellent seeing conditions (0.3-0.5 arcsec) at low airmass (1.02-1.08) together with solar-type telluric analogues.

Six individual exposures, comprising three AB pairs, were obtained for each grating setting, with wavelength solutions achieved from comparison $\mathrm{XeAr}$ arc datasets. From these, the observations covered 1.671-1.751 $\mu \mathrm{m}, 2.029-2.155 \mu \mathrm{m}$ and 2.140-2.265 $\mu \mathrm{m}$ at spectral resolutions of $3.8 \AA, 6.0 \AA$ and $6.0 \AA$ respectively, as measured from arc lines.

Telluric correction was achieved from a single $A B$ pair of spectroscopic datasets of early-G dwarfs observed at a similar airmass to $\mathrm{G} 23.96+0.15$, corrected for their spectral features using high resolution observations of the Sun which were adjusted to both the radial velocity and spectral resolution of the template stars.

The $2.09 \mu \mathrm{m}$ setup suffered from low-level variable structure which was accentuated upon flat-fielding, so that only the two other settings were flat-fielded. Consequently, the continuum $\mathrm{S} / \mathrm{N}$ achieved was $\sim 100$ for the $1.71 \mu \mathrm{m}$ setting and 150 for the $2.20 \mu \mathrm{m}$ setting for \#1, but only 60 for the $2.09 \mu \mathrm{m}$ setting. The continuum $\mathrm{S} / \mathrm{N}$ for sources \#2 and \#3NW was, at best, no greater than $40-50$.

\section{The ionizing star of $\mathrm{G} 23.96+0.15$}

\subsection{Near-IR spectral classification}

Hanson et al. (1996) developed a classification scheme for $\mathrm{O}$ stars from low resolution $K$-band spectroscopy. Classification of early to mid-O stars was achieved from the presence of C IV $2.08 \mu \mathrm{m}$ and $\mathrm{N}$ III $2.11 \mu \mathrm{m}$ emission lines, although it proved to be problematic to distinguish between late $\mathrm{O}$ and early $\mathrm{B}$ stars. This technique was extended to the $H$-band by Hanson et al. (1998) who highlighted the diagnostic role of the hydrogen $\mathrm{Br} 11$ $1.681 \mu \mathrm{m}$, He II $1.692 \mu \mathrm{m}$ and He I $1.700 \mu \mathrm{m}$ lines.

In Fig. 2 we present $H$-band and $K$-band spectroscopy of $\mathrm{G} 23.96+0.15$ \#1 together with MK classification $\mathrm{O}$ dwarfs from the high quality, medium resolution atlas of Hanson et al. (2005b). From visual inspection, a classification of O7-8 is estimated, in agreement with the low resolution $K$-band spectrum of \#1 from Hanson et al. (2002). However, we have also investigated the possibility of using the He II $1.692 \mu \mathrm{m} / \mathrm{He}$ I $1.700 \mu \mathrm{m}$ 

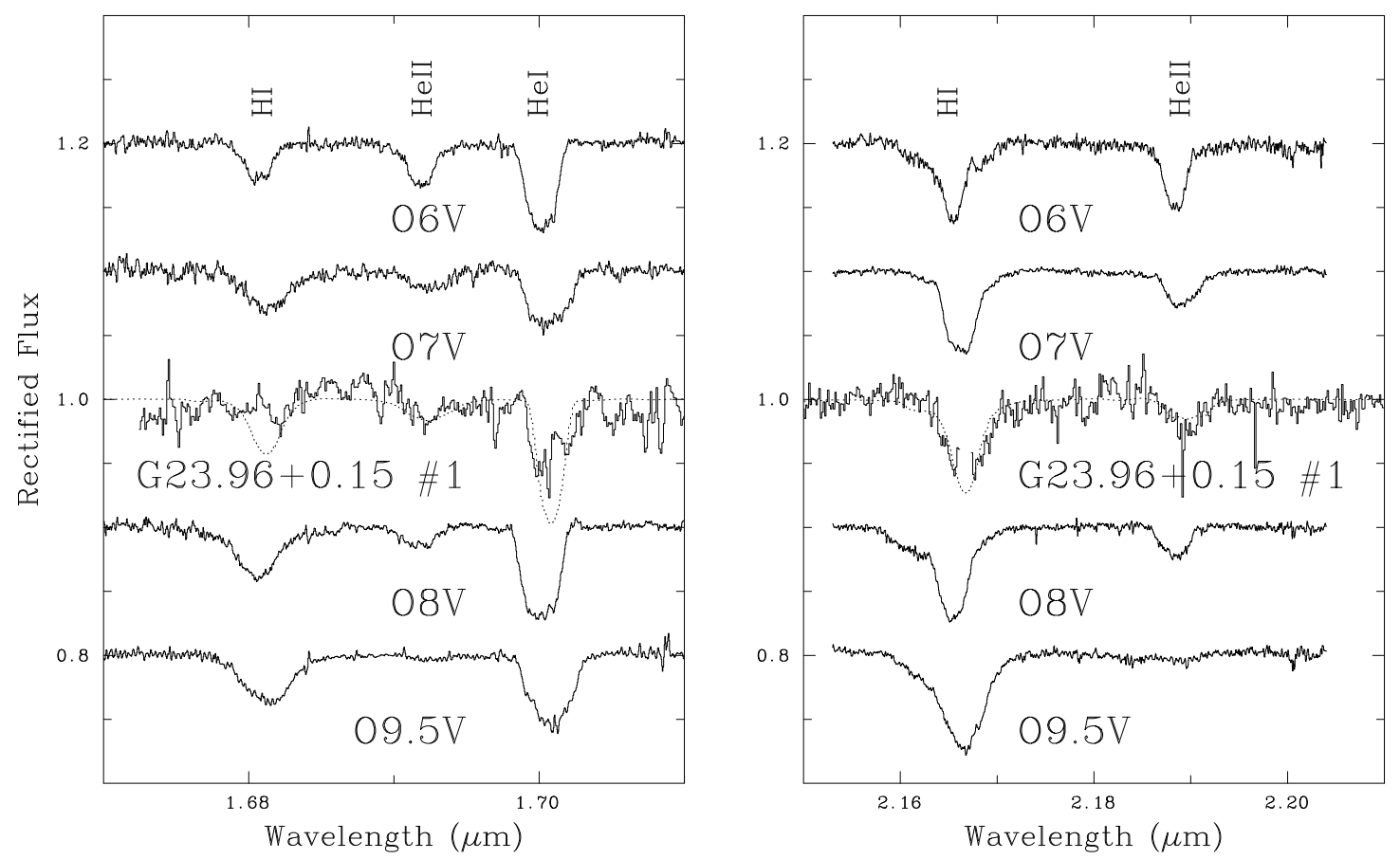

Fig. 2. $H$-band (left) and $K$-band (right) VLT/ISAAC spectroscopy of G23.96+0.15 \#1 together with MK template O6-9.5V stars from Hanson et al. (2005b), together with a spectral fit to \#1 (dotted line, see text).

and $\mathrm{He}$ II $2.189 \mu \mathrm{m} / \mathrm{Br} \gamma$ ratios for quantitative classification, independent of the metal lines in the $K$-band (see also Lenorzer et al. 2004; Repolust et al. 2005).

We have measured the equivalent widths of these lines for MK classification $\mathrm{O}$ stars from Hanson et al. (2005b), whose absorption line ratios are presented in Fig. 3 (excluding $\mathrm{Br} \gamma$ emission line supergiants). From this it is clear that the He II $1.692 \mu \mathrm{m} / \mathrm{He}$ I $1.700 \mu \mathrm{m}$ ratio provides an excellent classification diagnostic for $\mathrm{O}$ stars, especially dwarfs, while the He II $2.189 \mu \mathrm{m} / \mathrm{Br} \gamma$ ratio provides an additional constraint for late-type $\mathrm{O}$ dwarfs, providing the spectral resolution is sufficient to exclude any nebular contamination. Polynomial fits to equivalent width ratios of dwarf stars from Hanson et al. (2005b) are presented as a guide, excluding HD 37468 from the fit for which negligible He II absorption is observed. The $H$-band ratio inherently represents a superior diagnostic, although the $K$-band ratio has two advantages for late-type $\mathrm{O}$ dwarfs, namely a lower interstellar extinction plus $2.189 \mu \mathrm{m}$ is an intrinsically stronger He II line than $1.692 \mu \mathrm{m}$.

Unfortunately, the moderate $\mathrm{S} / \mathrm{N}$ of sources \#2 and \#3 prevented identification of spectral feaures beyond $\operatorname{Br} \gamma$ nebular emission. We present $H$ and $K$-band photometry of these sources in Table 1 together with absorption line measurements of $\mathrm{G} 23.96+0.15 \# 1$. From the lower $\mathrm{S} / \mathrm{N} H$-band ratio we infer a dwarf spectral type of $\mathrm{O}_{-0.5}^{+1}$ while the $K$-band diagnostic indicates $\mathrm{O} 7.5 \pm 0.5$. Therefore, we propose an $\mathrm{O} 7.5 \mathrm{~V}$ classification for source \#1.

\subsection{Stellar temperature of G23.96+0.15 \#1}

The current spectroscopic calibration of $\mathrm{O}$ stars would result in an effective temperature of $T_{\text {eff }}=36 \mathrm{kK}$ for an $07.5 \mathrm{star}$ (Martins et al. 2005). Instead, we have obtained a direct estimate of the stellar temperature from a comparison between the nearIR hydrogen and helium lines and synthetic spectra obtained with the CMFGEN model atmosphere code (Hillier \& Miller 1998).

CMFGEN solves the radiative transfer equation in the comoving frame, under the additional constraint of statistical equilibrium. The temperature structure is determined by radiative equilibrium. Since CMFGEN does not solve the momentum equation, a density or velocity structure is required. For the supersonic part, the velocity is parameterized with a classical $\beta$-type law, with an exponent of $\beta=1$ adopted. This is connected to a hydrostatic density structure at depth, such that the velocity and velocity gradient match at the interface. The subsonic velocity structure is set by a corresponding fully line-blanketed planeparallel TLUSTY model (v.200, see Lanz \& Hubeny 2003). The atomic model is similar to that adopted in Hillier et al. (2003), including ions from $\mathrm{H}, \mathrm{He}, \mathrm{C}, \mathrm{N}, \mathrm{O}, \mathrm{Ne}, \mathrm{Si}, \mathrm{S}, \mathrm{Ar}, \mathrm{Ca}$ and $\mathrm{Fe}$.

We have assumed a depth-independent Doppler profile for all lines when solving for the atmospheric structure in the comoving frame, while in the final calculation of the emergent spectrum in the observer's frame, we have adopted a uniform turbulence of $50 \mathrm{~km} \mathrm{~s}^{-1}$. Incoherent electron scattering and Stark broadening for hydrogen and helium lines are adopted. Finally, we convolve our synthetic spectrum with a rotational broadening profile.

For an adopted terminal wind velocity of $2000 \mathrm{~km} \mathrm{~s}^{-1}$, surface gravity of $\log g=4$ and abundance ratio of $\mathrm{He} / \mathrm{H}=0.1$ by number, we varied the stellar radius and (non-clumped) mass-loss rate until a reasonable match to the He II $1.692 \mu \mathrm{m}$, He I $1.700 \mu \mathrm{m}, \mathrm{Br} \gamma$ and He II $2.189 \mu \mathrm{m}$ was achieved. An acceptable fit was achieved for $T_{\text {eff }}=38 \pm 1 \mathrm{kK}, \log \dot{M} /\left(M_{\odot} \mathrm{yr}^{-1}\right)=$ $-6.3 \pm 0.2$ and $v \sin i \sim 220 \pm 30 \mathrm{~km} \mathrm{~s}^{-1}$, as shown in Fig. 2. Nebular contamination is significant for He I $1.700 \mu \mathrm{m}$ and high members of the hydrogen Brackett series. For an adopted mass of $30 M_{\odot}$, a radius of $9.2 R_{\odot}$ results, which is representative of normal Milky Way mid-O dwarfs (e.g. Repolust et al. 2005). We defer any further discussion of the physical properties of 

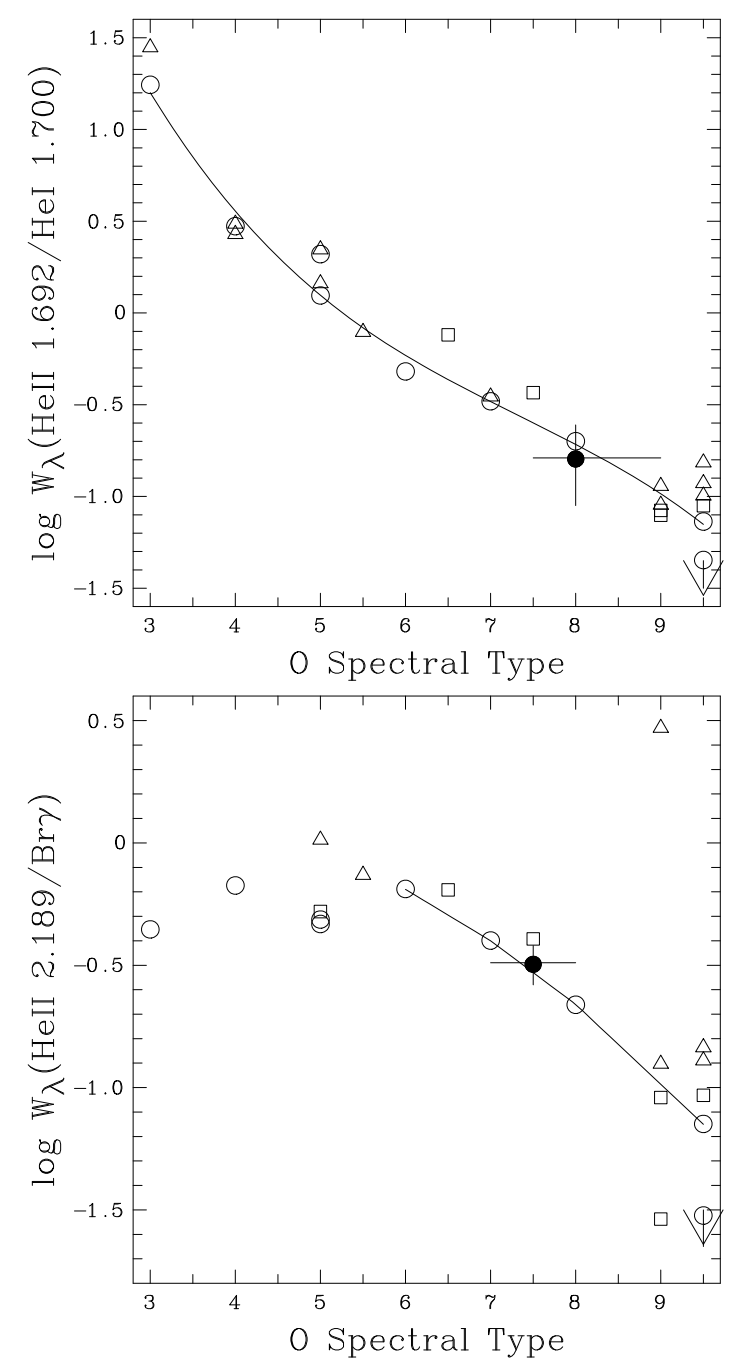

Fig. 3. (Top) Equivalent width ratio of He II $1.692 \mu \mathrm{m} / \mathrm{He}$ I $1.700 \mu \mathrm{m}$ versus subtype for $\mathrm{O}$ dwarfs (circles), giants (squares) and supergiants (triangles) from Hanson et al. (2005a) and G23.96+0.15 \#1 (filled circle) together with a polynomial fit to dwarfs as a guide (solid line); (bottom) as above for ratio of $\mathrm{He}$ II $2.189 \mu \mathrm{m} / \mathrm{Br} \gamma$.

G23.96+0.15 \#1 until the next section where its distance is considered.

\section{Distance and extinction to $\mathrm{G} 23.96+0.15$}

Distances to Galactic H II regions are typically obtained from kinematic methods, using an adopted rotation curve (Brand \& Blitz 1993) and Solar galactocentric distance (8 kpc, Reid 1993). Following this approach the near (far) kinematic distance to $\mathrm{G} 23.96+0.15$ is $4.7 \mathrm{kpc}(10.0 \mathrm{kpc})$ based upon the LSR velocity of $79.3 \mathrm{~km} \mathrm{~s}^{-1}$ from Wink et al. (1983), suggesting a distance of $4.2 \mathrm{kpc}$ from the Galactic Centre for G23.96+0.15. LSR velocities from $\mathrm{NH}_{3}$ (Churchwell et al. 1990), CS (Plume et al. 1992) and $\mathrm{H} 76 \alpha$ recombination lines observations (Kim \& Koo 2001) agree with Wink et al. (1983) to within $\pm 1 \mathrm{~km} \mathrm{~s}^{-1}$.

One would expect $M_{K}=-3.9 \mathrm{mag}$ for $\mathrm{G} 23.96+0.15$ \#1 (O7.5V) from the Conti et al. (2008) absolute visual magnitude-spectral type calibration, together with the intrinsic colours of Martins \& Plez (2006). We may correct its observed $K$-band magnitude for extinction using $A_{K}=1.82_{-0.23}^{+0.30} E_{H-K}$ (Indebetouw et al. 2005) and $(H-K)_{0}=-0.10 \mathrm{mag}$, from
Table 2. Physical properties of G23.96+0.15 \#1 from our spectral analysis, based upon either the spectroscopic (top) or kinematic (bottom) distances. Mass estimates are approximate since we adopt $\log g=4.0$. The predicted Lyman continuum ionizing flux $\left(Q_{0}\right)$ is shown together with lower limits obtained from the $21 \mathrm{~cm}$ radio observations of Kim \& Koo (2001, $\left.Q_{0}^{\mathrm{KK} 01}\right)$.

\begin{tabular}{rrrrrrrr}
\hline \hline$T_{\text {eff }}$ & $\log L$ & $M$ & $\log \dot{M}$ & $\log Q_{0}$ & $\log Q_{0}^{\mathrm{KK} 01}$ & $M_{K}$ & $\begin{array}{c}d \\
\mathrm{kK}\end{array}$ \\
$L_{\odot}$ & $M_{\odot}$ & $M_{\odot} \mathrm{yr}^{-1}$ & $\mathrm{~s}^{-1}$ & $\mathrm{~s}^{-1}$ & $\mathrm{mag}$ & $\mathrm{kpc}$ \\
\hline 38 & 5.2 & 30 & -6.3 & 48.8 & $\geq 48.1$ & -3.9 & 2.5 \\
38 & 5.8 & 130 & -5.8 & 49.4 & $\geq 48.6$ & -5.5 & 4.7 \\
\hline
\end{tabular}

which $A_{K}=2.33 \pm 0.3$ mag, suggesting a distance modulus of $12.0_{-0.4}^{+0.3}$, i.e. a distance of $2.5 \pm 0.4 \mathrm{kpc}$. For a representative scatter of $\pm 0.5 \mathrm{mag}$ in the absolute magnitude calibration of $\mathrm{O}$ stars, a spectroscopic distance in the range $2-3.2 \mathrm{kpc}$ would be implied, placing G23.96+0.15 at a distance of $5.8 \pm 0.5 \mathrm{kpc}$ from the Galactic Centre.

Martín-Hernández et al. (2002) have previously estimated $A_{K}=2.0 \mathrm{mag}$ for $\mathrm{G} 23.96+0.15$ based upon HI recombination lines observed in Infrared Space Observatory (ISO) spectroscopy. We independently confirm this result from the observed ISO $\operatorname{Br} \alpha / \operatorname{Br} \beta$ ratio, although the ISO pointing was offset by $\geq 10$ arcsec from the source peak, and so measured fluxes need to be treated with caution (Peeters et al. 2002).

In common with other studies of $\mathrm{H}$ II regions, we find a spectroscopic distance to $\mathrm{G} 23.96+0.15$ that is substantially lower than kinematic distances (e.g. Blum et al. 2001; Figueredo et al. 2008). The two distances could be reconciled if \#1 were substantially more luminous than typical mid-O stars, with $M_{K}=$ $-5.5 \mathrm{mag}$, although this would require the star to be extremely massive if it is single. This is highlighted in Table 2, where physical properties of $\mathrm{G} 23.96+0.15 \# 1$ for each of the alternate distances are presented. Of course, \#1 could be an unresolved equal mass binary (or compact star cluster). Such a source would appear $\sim 0.75(0.3)$ mag brighter in the $K$-band than a single 07.5 star, owing to the contribution of its companion(s) ${ }^{1}$. A binary/cluster scenario would help to alleviate the spectroscopic and kinematic distance discrepancy, although in the cluster case, stellar absorption lines from the $\mathrm{O}$ star would be diluted by the continuum light from lower mass cluster members, which does not appear to be the case for G23.96+0.15 \#1 (recall Fig. 2). Similar remarks apply for G29.96-0.02, for which a spectroscopic distance of $\sim 3 \mathrm{kpc}$ would be expected for a single O4 dwarf using near-IR photometry from Pratap et al. (1999), compared with a kinematic distance of $7.4 \mathrm{kpc}$ (Fish et al. 2003).

Kim \& Koo (2001) estimated an ionizing output of $10^{48.8} \mathrm{ph} \mathrm{s}^{-1}$ for $\mathrm{G} 23.96+0.15 \mathrm{~A}$ from their $21 \mathrm{~cm}$ radio continuum observations, which is equivalent to an $\mathrm{O} 7$ dwarf (Conti et al. 2008), albeit based upon an assumed distance of $6 \mathrm{kpc}$. If one was to adjust the distance to $4.7(2.5) \mathrm{kpc}$, a reduced Lyman continuum output would be obtained, representative of an O8 (O9.5) star (Table 2). The measured $21 \mathrm{~cm}$ flux, $\sim 0.75 \mathrm{dex}$ lower than our indirect estimate, is merely a lower limit since it may be partially optically thick to bremstrahlung radiation and a fraction of the ionizing photons are likely to be absorbed by dust (either within the H II region or circumstellar cocoon).

The near-IR colours of sources \#2 and \#3NW are consistent with being physically associated with $\mathrm{G} 23.96+0.15$ \#1 (\#3SE is likely a foreground source). For the spectroscopic distance of $2.5 \mathrm{kpc}$, they are probable early B dwarfs, at projected

\footnotetext{
1 On the basis of a Salpeter-like Initial Mass Function for high mass stars.
} 
distances of $0.02 \mathrm{pc}(\# 2)$ and $0.05 \mathrm{pc}(\# 3 \mathrm{NW})$ from \#1. In common with indirect dust and gas diagnostics for other UCHII regions (Okamoto et al. 2003), we find evidence that G23.96+0.15 likely hosts multiple massive stars (see also Hunter et al. 2004). For the kinematic (near) distance of $4.7 \mathrm{kpc}$, sources \#2 and \#3NW would possess absolute magnitudes typical of late $\mathrm{O}$ stars, and so would also most likely be unresolved sub-clusters within the UCHII region, at projected distances of $0.035-0.1 \mathrm{pc}$ from \#1.

\section{Conclusions}

We show that $\mathrm{G} 23.96+0.15$ provides a second UCHII region whose ionizing star (\#1) is accessible to high $\mathrm{S} / \mathrm{N}$, medium resolution near-IR spectroscopy, complementing the higher ionization G29.96-0.02 source. We provide a quantitative nearinfrared classification scheme for $\mathrm{O}$ stars, which has potential application for other visibly obscured $\mathrm{O}$ dwarfs in the Milky Way, from which a near-IR $07.5 \mathrm{~V}$ classification is obtained for $\mathrm{G} 23.96+0.15 \# 1$. We obtain a stellar temperature of $T_{\text {eff }}=38 \mathrm{kK}$ for $\mathrm{G} 23.96+0.15 \# 1$ and infer a spectroscopic distance of $2.5 \mathrm{kpc}$ for a single star origin. This is substantially smaller than the (near) kinematic distance of $4.7 \mathrm{kpc}$, in common with other obscured H II regions (e.g. Blum et al. 2001; Figueredo et al. 2008), although an unresolved binary or cluster nature would help to alleviate this discrepancy. Two fainter sources are also likely earlytype members of the UCHII region. The availability of spectral properties for $\mathrm{G} 23.96+0.15$ should help empirically address the "inverse problem" of obtaining the ionizing $\mathrm{O}$ stars for other UCHII regions based solely upon mid-IR nebular characteristics.

Acknowledgements. Many thanks to Margaret Hanson for providing her $H$-band image of G23.96+0.15 and intermediate resolution near-infrared spectral atlas. This publication makes use of data products from 2MASS, which is a joint project of the University of Massachusetts and the IPAC/CalTech, funded by the NASA and the NSF, and is, in part, based upon archival observations from UKIRT which is operated by the Joint Astronomy Centre on behalf of the Science and Technology Facilities Council (STFC) of the UK. J.P.F. acknowledges financial support from the STFC.

\section{References}

Bik, A., Kaper, L., Hanson, M. M., \& Smits, M. 2005, A\&A, 440, 121 Blum, R. D., Damineli, A., \& Conti, P. S. 2001, AJ, 121, 3149 Brand, J., \& Blitz, L. 1993, A\&A, 275, 67

Churchwell, E., Walmsley, C. M., \& Cesaroni, R. 1990, A\&AS, 83, 119

Conti, P. S., Crowther, P. A., \& Leitherer, C. 2008, From Luminous Hot Stars to Starburst Galaxies, Cambridge Astrophysics Series, 45 (Cambridge: CUP) Figueredo, E., Blum, R. D., Damineli, A., Conti, P. S., \& Barbosa, C. L. 2008, AJ, 136, 221

Fish, V. L., Reid, M. J., Wilner, D. J., \& Churchwell, E. 2003, ApJ, 587, 701

Hanson, M. M., Conti, P. S., \& Rieke, M. J. 1996, ApJS, 107, 281

Hanson, M. M., Rieke, G. H., \& Luhman, K. L. 1998, AJ, 116, 1915

Hanson, M. M., Luhman, K. L., \& Rieke, G. H. 2002, ApJS, 138, 35

Hanson, M. M., Puls, J., \& Repolust, T. 2005a, in Proc. IAU Symp. 227, Massive Star Birth: a crossroads of astrophysics ed. R. Cesaroni, M. Felli, E. Churchwell, \& M. Walmsley (Cambridge: CUP), 376

Hanson, M. M., Kudritzki, R.-P., Kenworthy, M. A., Puls, J., \& Tokunaga, A. T. 2005b, ApJS, 161, 154

Hillier, D. J., \& Miller, D. L. 1998, ApJ, 496, 407

Hillier, D. J., Lanz, T., Heap, S. R., et al. 2003, ApJ, 588, 1039

Hunter, T. R., Zhang, Q., \& Sridharan, T. K. 2004, ApJ, 606, 929

Indebetouw, R., Mathis, J. S., Babler, B. L., et al. 2005, ApJ, 619, 931

Kim, K.-T., \& Koo, B.-C. 2001, ApJ, 549, 979

Lenorzer, A., Mokiem, M. R., de Koter, A., \& Puls, J. 2004, A\&A, 422, 275

Martín-Hernández, N. L., Peeters, E., Morisset, C., et al. 2002, A\&A, 381, 606 Martins, F., \& Plez, B. 2006, A\&A, 457, 637

Martins, F., Schaerer, D., \& Hillier, D. J. 2005, A\&A, 436, 1049

Moorwood, A., Cuby, J.-G., Biereichel, P., et al. 1998, ESO Messenger, 94, 7

Morriset, C., Schaerer, D., Bouret, J.-C., \& Martins, F. 2004, A\&A, 415, 577

Okamoto, Y. K., Kataza, H., Yamashita, T., et al. 2003, ApJ, 584, 368

Peeters, E., Martín-Hernández, N. L., Damour, F., et al. 2002, A\&A, 381, 571

Plume, R., Jaffe, D. T., \& Evans, N. J. 1992, ApJS, 78, 505

Pratap, P., Megeath, S. T., \& Bergin, E. A. 1999, ApJ, 517, 799

Prescott, M. K. M., Kennicutt, R. C. Jr., Bendo, G. J., et al. 2007, ApJ, 668, 182 Reid, M. J. 1993, ARA\&A, 31, 345

Repolust, T., Puls, J., Hanson, M. M., et al. 2005, A\&A, 440, 261

Roche, R. F., et al. 2002, Proc. SPIE, 4841

Watson, A. M., \& Hanson, M. M. 1997, ApJ, 490, L165

Wink, J. E., Wilson, T. L., \& Bieging, J. H. 1983, A\&A, 127, 211

Wood, D. O. S., \& Churchwell, E. 1989, ApJS, 69, 831

Zinnecker, H., \& Yorke, H. W. 2007, ARA\&A, 45, 481 